\title{
Intraoral appliances for temporomandibular disorders: what we know and what we need to know
}

\author{
Harold F. Menchel ${ }^{1}$, Charles S. Greene ${ }^{2}$, Kevin D. Huff ${ }^{3}$ \\ ${ }^{1}$ Department Orofacial Pain, Nova Southeastern School of Dental Medicine, Davie Florida, USA; ${ }^{2}$ Department of Orthodontics, University of \\ Illinois College of Dentistry, Chicago, USA; ${ }^{3}$ Private Practice, Dover, Ohio, USA \\ Contributions: (I) Conception and design: HF Menchel; (II) Administrative support: CS Greene; (III) Provision of study materials or patients: None; \\ (IV) Collection and assembly of data: None; (V) Data analysis and interpretation: None; (VI) Manuscript writing: All authors; (VII) Final approval of \\ manuscript: All authors. \\ Correspondence to: Harold F. Menchel, DMD. Nova Southeastern School of Dental Medicine, 3301 College Avenue, Ft. Lauderdale, Florida 33314, \\ USA. Email: hmenchel@nova.edu.
}

\begin{abstract}
Oral appliances (OAs) have been a part of the clinical protocols used by many dentists over the past 100 years for treating patients with temporomandibular disorders (TMDs). However, in a field filled with many conceptual and clinical controversies, these devices remain near the top of the list of controversial topics. There are multiple designs proposed for making OAs, and there are disagreements about when and how they should be utilized. There are also theoretical arguments about what they are intended to do therapeutically. However, the most significant point of contention is whether OAs should be regarded as a first phase of TMD treatment, to be followed by a second phase of major dental procedures to realign the condylar position as well as the occlusal relationships. Alternatively, within the past 50 years many researchers have established an evidence base supporting a more conservative application of OAs in which they are an orthotic modality intended to produce relief from pain and improvement in jaw function-after which they may be removed or used to respond to symptomatic flareups. This paper discusses what we currently know, and what more we need to know, in order to deal with this confusing topic in the best interest of managing various categories of TMD patients. It concludes with a proposal for future research directions which may help to clarify some of these issues.
\end{abstract}

Keywords: Oral appliances (OAs); clinical indications; evidence base

Received: 31 July 2020; Accepted: 06 January 2021; Published: 30 March 2021.

doi: $10.21037 /$ fomm-20-49

View this article at: http://dx.doi.org/10.21037/fomm-20-49

\section{Introduction}

Oral appliances (OAs) have been used by many dentists over the past century to treat various forms of temporomandibular disorders (TMDs) (1). While initially there was no research support for using OAs, they have been subjected to a huge variety of controlled and uncontrolled studies since the second half of the $20^{\text {th }}$ century. Perhaps it is not surprising that this variety of clinical studies, systematic reviews, and even meta-analyses has produced a widely divergent set of results, thereby producing controversy within the dental profession about the true value of these appliances (2) (see Table 1).

Consequently, we find ourselves today in the situation of knowing some things about OAs, including how they can be designed, when they might best be used, and what kind of clinical results are likely to be obtained under various conditions. Favorable outcomes of decreased pain and improved mouth opening are the most commonly reported results from both academic studies as well as clinical experiences. Of course, even these points are subject to dispute; however, in this paper the authors intend to present the current evidence to support each of these clinical outcome observations. 
Table 1 Comparison of recent studies on the efficacy of OA therapy for TMD (3-16)

\begin{tabular}{|c|c|c|c|c|}
\hline Study & Type & OAs used & Diagnoses & Comments \\
\hline Riley et al. 2020 & $\begin{array}{l}\text { Systematic review } \\
\text { of RCTs }\end{array}$ & Multiple OAs & Multiple & $\begin{array}{l}\text { No significant improvement in pain or reduced tooth } \\
\text { wear }\end{array}$ \\
\hline $\begin{array}{l}\text { Katayan et al. } \\
2014\end{array}$ & RCT & $\begin{array}{l}\text { Upper stabilization } \\
\text { OA }\end{array}$ & $\begin{array}{l}\text { Multiple excluded } \\
\text { patients with } \\
\text { parafunction }\end{array}$ & $\begin{array}{l}\text { OA has no benefit over counseling in pain relief } \\
\text { or improvement of function than counseling and } \\
\text { exercises over } 6 \text { months }\end{array}$ \\
\hline $\begin{array}{l}\text { Fricton, Look } \\
\text { et al. } 2010\end{array}$ & $\begin{array}{l}\text { Systematic review } \\
\text { and meta-analysis }\end{array}$ & $\begin{array}{l}\text { Hard stabilization } \\
\text { OAs, compared } \\
\text { to non occluding } \\
\text { appliances }\end{array}$ & Multiple & $\begin{array}{l}\text { Hard stabilization OAs when adjusted properly have } \\
\text { good evidence of modest efficacy in the treatment of } \\
\text { TMJD pain to non occluding appliances }\end{array}$ \\
\hline Costa et al. 2015 & $\mathrm{RCT}$ & Stabilization OA & $\begin{array}{l}\text { Multiple psychological } \\
\text { aspects }\end{array}$ & $\begin{array}{l}\text { Minimally invasive strategies could provide an } \\
\text { improvement in the psychological aspects of } \\
\text { temporomandibular disorder patients, and the use of } \\
\text { an occlusal splint seems to hasten the manifestation } \\
\text { of these effects }\end{array}$ \\
\hline $\begin{array}{l}\text { Giannakopoulos } \\
\text { et al. } 2016\end{array}$ & RCT & $\begin{array}{l}\text { Hard acrylic } \\
\text { thermoplastic, and } \\
\text { prefabricated OAs for } \\
\text { short term use }\end{array}$ & Multiple & $\begin{array}{l}\text { Equal efficacy for hard Michigan splint and } \\
\text { thermoplastic in symptom improvement vs. } \\
\text { prefabricated OA }\end{array}$ \\
\hline $\begin{array}{l}\text { Qvintus et al. } \\
2015\end{array}$ & $\mathrm{RCT}$ & Stabilization OA & & $\begin{array}{l}\text { "The findings of this study did not show stabilisation } \\
\text { splint treatment to be more effective in decreasing } \\
\text { facial pain than masticatory muscle exercises and } \\
\text { counselling alone in the treatment of TMD-related } \\
\text { facial pain over a 1-year follow-up" }\end{array}$ \\
\hline $\begin{array}{l}\text { Wahkund et al. } \\
2015\end{array}$ & $\mathrm{RCT}$ & Stabilization OA & Multiple & $\begin{array}{l}\text { "The findings suggest that, for adolescents with } \\
\text { TMD pain, use of standardized clinical treatment } \\
\text { with OA is more effective than RT on self-evaluation } \\
\text { of treatment improvement." }\end{array}$ \\
\hline Pficer et al. 2017 & $\begin{array}{l}\text { Meta-analysis of } \\
\text { RCTs }\end{array}$ & Stabilization OA & $\begin{array}{l}\text { Subgroup analysis } \\
\text { according to DC/TMD }\end{array}$ & $\begin{array}{l}\text { SS presented short term benefit for TMD patient } \\
\text { with muscle pain. Long term benefit was not shown } \\
\text { compared to non-OA }\end{array}$ \\
\hline Rosar 2017 & $\begin{array}{l}\text { Clinical trial not } \\
\text { randomized }\end{array}$ & Stabilization OA & $\begin{array}{l}\text { No diagnosis other than } \\
\text { TMD }\end{array}$ & $\begin{array}{l}\text { Short term use had a positive effect on bite force } \\
\text { TMD symptoms, sleep quality, and salivary cortisol } \\
\text { levels in patients with SB }\end{array}$ \\
\hline
\end{tabular}

Notice the lack of standardization in the studies. This is particularly true in regard to the diagnoses used, inclusion and exclusion data, and no consistent design of appliance protocols. OA, oral appliance; TMD, temporomandibular disorder; RCT, randomized clinical trial. 
At the same time, we have to admit that there are things that we do not know about OAs, including exactly what the mechanisms are that explain how they might or might not work for certain TMD conditions. Also, the longterm impact of continuous OA wear remains a subject of deep controversy because such wear may produce major changes in occlusal and craniomandibular relationships. Furthermore, because there are so many physical designs of OAs, it is not clear which kind is most appropriate for treating any particular TMD condition as defined within the Diagnostic Criteria for Temporomandibular Orders (DC/TMD) (17). So, much remains to be done in order to answer that question. Therefore, this paper concludes by proposing a standardized study protocol that could at least provide some partial answers to that important question.

As concerns the DC/TMD diagnoses, there is a case to made from the current evidence that OAs may be most effective in management of two common TMD conditions: (I) regional myofascial pain; and (II) inflammatory TM joint disease, especially the pain associated with degenerative joint disease (DJD) (18). OAs are a reasonable therapeutic modality because they provide a conservative and relatively reversible treatment approach that is non-pharmaceutical, with minimal adverse effects when used with accepted appliance designs and protocols. They do not typically require invasive treatment, and they generally do not produce permanent anatomic changes in the stomatognathic system. While additional therapeutic modalities such as physiotherapy, cognitive behavioral therapy, patient education, and pharmacotherapy all play important roles in the non-surgical management of TMDs, OAs should definitely be considered among the pragmatic first line therapeutic options that may be prescribed by orofacial pain specialists as well as by appropriately trained general dentists.

\section{What does the recent literature say about the value of OAs?}

Unfortunately, to date, there is only weak to moderate evidence from randomized clinical trials (RCTs), metaanalyses, and systematic reviews to support the use of OAs for TMD pain management. As shown in Table 1, these weaknesses are due to a multiplicity of factors: inclusion of multiple DC/TMD diagnoses within some studies, multiple splint designs, different criteria for outcome success or failure, and lack of controls. In addition, it is also difficult to assess compliance of the subject group, what kinds of medications they are taking, and other behavioral changes experienced by the patients during therapy. For instance, in a recent Cochrane search there were no studies where subjects were limited to OA therapy only, with no medication, no change in diet, and assurances of continuing present behavior.

However, the main weakness in the current evidence base is due in large part to the continuing decades old controversy about what OAs are intended to do. More than likely, they are type of conservative therapy within the biopsychosocial model of TMD therapy meant to manage pain and dysfunction in TMD patients. These patients have multifactorial contributing etiologic issues including both Axis I (biophysical) and Axis II (psychosocial) DC/TMD categories of susceptibility and perpetuating factors. Based on this model, most current treatment guidelines suggest a conservative management strategy for the management of all but the most severe cases (19).

Nevertheless, some clinicians believe that OAs should be regarded as devices that are intended as a preliminary or diagnostic step prior to definitive dental therapy. Within that conceptual framework, an OA is used to modify joint position and anatomy, evaluate vertical dimension of occlusion, "deprogram" the patient so that irreversible occlusal modification can be performed in "phase II" treatment, or other mechanistic protocols with minimal consideration for psychosocial contributions (20-22). Those ascribing to this philosophy of TMD management assume that an ideal condylar treatment position can be determined by bimanual manipulation, deprogramming and even TENS fatiguing of muscles. We now know that the condyle can function physiologically in many positions (23). With the increasing lack of evidence supporting that latter view of TMD, future research directions need to concentrate on the potential for the conservative therapeutic value of OAs. It is the opinion of the authors that this is likely to be the most productive avenue for research.

\section{Probable mechanisms of $\mathrm{OA}$ therapy}

Based on current understanding of the multifactorial etiology for TMD signs and symptoms, some probable mechanisms for the observed positive effect of OA therapy may be the following:

\section{Reduced joint loading}

OAs decrease mechanical stress and joint inflammation by changing the load applied to the TMJs. In fact, there are a number of studies in vivo, in vitro, and based on computer 
modeling that consistently show reduced force to TMJs with OAs, particularly regarding OAs with posterior occlusal support (2). Since mechanical stress on joints has been shown to cause inflammation $(24,25)$, it therefore could be inferred that OAs reducing joint load should result in decreased inflammation. A recent RCT (26) comparing post arthrocentesis patients wearing OAs with the controls not wearing an OA, showed improvement in symptoms following the intracapsular procedure; however, there were no differences found in concentrations of 3 inflammatory mediators after 3 and 6 months. Although these results are negative, they encourage future research with this model to be repeated with assay of other inflammatory mediators which may be reduced as part of the healing process.

\section{Behavioral changes may explain both muscular and joint responses to wearing an $\mathrm{OA}$}

Associated behavioral changes may explain both muscular and joint responses to OA therapy. There are well-designed studies reporting behavioral modification with OAs $(17,27)$. These findings fit well within the biopsychosocial model of care for TMDs. Furthermore, Findings from PSG sleep lab studies indicate that that OAs may reduce bruxism on a short-term basis $(28,29)$. OAs change proprioception, which increases brain modulation of muscle activity for 2-6 weeks as a protective mechanism to something foreign. During this time, especially when augmented with other interventions like cognitive behavior therapy, counseling, anti-inflammatory medications, and physiotherapy, one might expect to see a variety of positive responses as TMD signs and symptoms improve. Then the OA may become a "crutch" to stabilize the system (30). After the initial reduction in bruxism the CNS accommodates to the appliance and engrams return to normal parafunction for those patients.

\section{Placebo effect}

As a general medical patient management consideration, it has been suggested that the placebo effect of an intervention has a significant positive effect on the outcome and can often persuade the clinician that both the diagnosis and treatment are correct. This may account for the perception by many dentists who either do or do not spend time meticulously adjusting "nightguards", that the appliances they provide are highly effective in treating TMD, even without a clear diagnosis. OAs provide a physical method to justify patient contact appointments for patient education, counseling, and other doctor-patient interactions that collectively can contribute to producing positive responses. Furthermore, patients who have been effectively treated with OA therapy tend to depend on long-term wear of the OA to prevent pain from recurring; there is, however, no evidence to support this strong patient belief. It is not clear from the literature on OAs whether patients with certain TMD diagnoses should continue OA wear after their symptoms have abated. One study showed that after patients ceased OA wear for 15 days after 6 months of night wear that their pain recurred (30). Further studies are needed.

\section{Regression to the mean}

Clinical experience and many no-treatment observational studies suggest that levels of chronic pain with no objective disease findings tend to reduce without therapy over time to about $50 \%$ of maximum levels, which may be due to psychological or physical adaptation or both. In medical pain management, this is commonly referred to as "regression to the mean" (31). OAs may simply enable or expedite that process.

The combination of all these diverse theories of how OAs may work simply emphasizes the need in future OA studies to identify, distinguish, and separate DC/TMD diagnoses in the subjects as well as determining the length of the study for improved accuracy of information. Researchers must accept the fact that "TMD" is not the name of a diagnosis, but rather it is the name of a category of clinical problems affecting the stomatognathic system. Therefore, specific diagnoses must be established clinically in order to gain any data that is clinically relevant to the management of any particular type of TMD.

\section{Most common $\mathrm{OA}$ designs}

OAs can be directive or non-directive (permissive) in design. With a directive OA there are usually tooth indentations or "guides" on the cameo occluding surface of the OA to hold the opposing teeth in a given position depending on the treatment philosophy of the dentist (32). With non- directive OAs, the biting surface is relatively flat relative to the occlusal plane and allows freedom of excursive movement without eccentric interference. There is little evidence to support the benefit of directive splints that "lock" the patient in one position; furthermore, these OAs can actually increase muscle activity and bruxism (28). The exception to this is the anterior repositioning $\mathrm{OA}$, as 


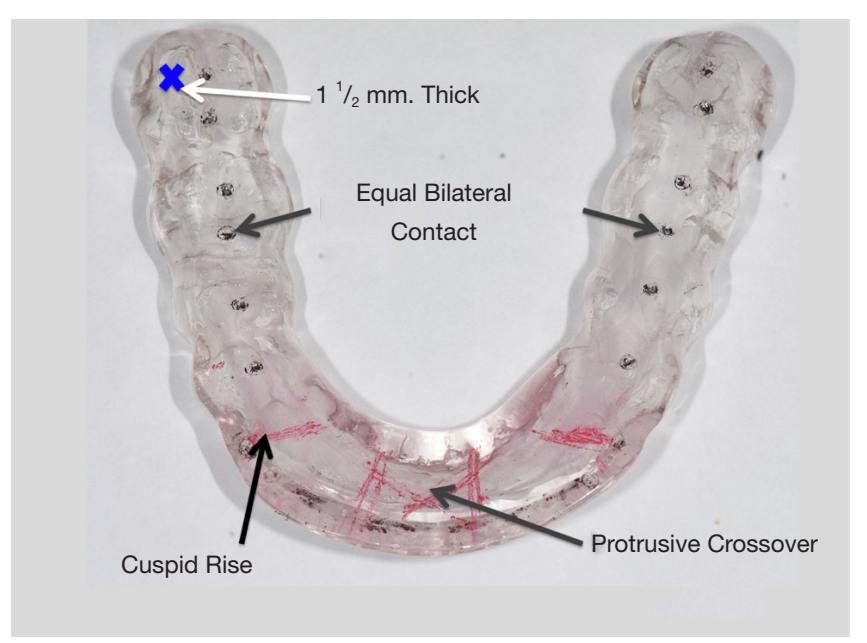

Figure 1 Example of lower adjusted flat plane full coverage hard acrylic OA. This example FP is adjusted optimally to even bilateral contact (black marks) and shallow anterior guidance including cuspid rise, protrusive, and cross-over adjusted in red. The crossover contacts keep the upper cuspids from falling off the lingual of the $\mathrm{OA}$ as the contacts are transitioned to the upper incisors. The minimum recommended thickness in the 2 nd molar area is $1.5 \mathrm{~mm}$ to assure minimum perforation when adjusting the OA. OA, oral appliance.

discussed below, which is used for a specific purpose.

In general, the most commonly used OAs can be grouped into five categories. They are utilized by clinicians who cite various treatment philosophies. OAs of each type are fabricated by a variety of materials according to clinician preference and perceived patient comfort, all of which add variables that must be accounted for in future research. Some examples include hard polymethylmethacrylate acrylic, hard and soft thermoplastic materials of varying thicknesses that may or may not be laminated with hard acrylic, milled resin, and even cast metal. Anecdotally, the more rigid the $\mathrm{OA}$ is, the more predictable the outcome (2). In general, the common appliance design categories are:

(I) Full-coverage flat plane stabilization appliance;

(II) Mandibular repositioning appliance;

(III) Anterior-only contact appliance;

(IV) Posterior bilateral platform appliance with no anterior contact;

(V) Neuromuscular full coverage appliances with joint position determined by electronic instrumentation.

Full coverage flat plane hard stabilization appliance (FP) This is the most common full coverage OA with a flat occluding surface, and it features equal bilateral contacts adjusted with or without anterior guidance. This design has been proven to be the safest, especially for long term wear, providing mechanical protection to the joints and avoiding permanent occlusal changes (see Figure 1).

\section{Mandibular repositioning OAs [anterior repositioning appliances (ARAs)]}

These appliances are also full coverage and usually fabricated for the maxillary arch, but they can also be fabricated for the mandibular arch (see Figure 2). Mandibular versions tend to have excessive labial bulk and may result in less patient compliance (Figure 3). There is a directive anterior ramp on the ARAs, but the posterior surfaces are flat allowing some freedom of movement (although less than a typical FP). The ramp on the anterior positions the mandible into a protrusive position.

ARAs are indicated in patients with diagnosis of disc displacements with reduction under certain conditions, such as disc displacements with reduction with intermittent locking (33). In these patients, anecdotal observations suggest that locking occurs most commonly upon awakening from sleep. It also has been reported that ARAs can be helpful in preventing clicking patients from progressing to disc displacement without reduction in patients with increasing occasional locking (34). There is a cautionary consensus that ARAs should be used short term to avoid permanent occlusal changes (posterior open bite). ARAs, therefore, have potential therapeutic advantages; however, there is no support for using this protrusive position as a template for permanent occlusal therapy.

\section{Anterior only contacting OAs (ACs)}

The configuration of anterior only contacting OAs (ACs) allows only the anterior teeth to touch on a flat plane perpendicular to the ala-tragus line in a sagittal view and the horizon in the frontal plane, or when facing the patient. This plane can be small with only the central incisors touching or may be extended to the cuspids. These appliances have been reported to reduce masseter hyperactivity when clenching (35). According to some advocates of this design, these OAs are proposed to be more effective in the treatment of migraine and tension headache than FPs. However, the overwhelming evidence is that there are no specific TMD diagnoses that show improved outcomes with these appliances (36). The concept of how ACs may work is that the narrower the platform the less elevator muscle activity can be generated., although other 

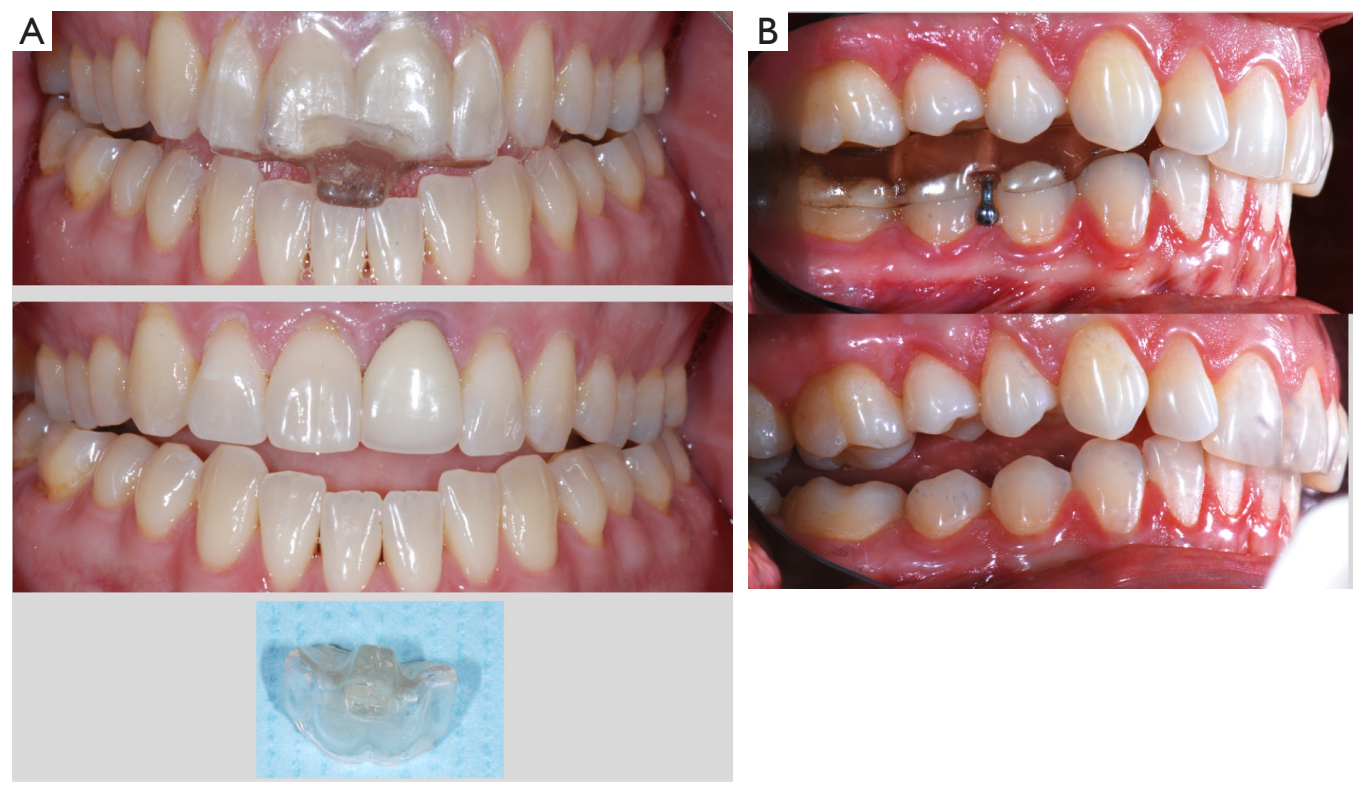

Figure 2 Examples of occlusal changes associated with oral appliances. (A) Anterior contacting appliance. This appliance was worn for 4 years only during sleep. The opposing adaptive occlusal changes are impressive. (B) Posterior contacting OA. This OA (Gelb appliance) was worn in a 24-year-old woman for 2 years with continuous wear (daytime and nighttime). Notice the occlusal changes. OA, oral appliance.

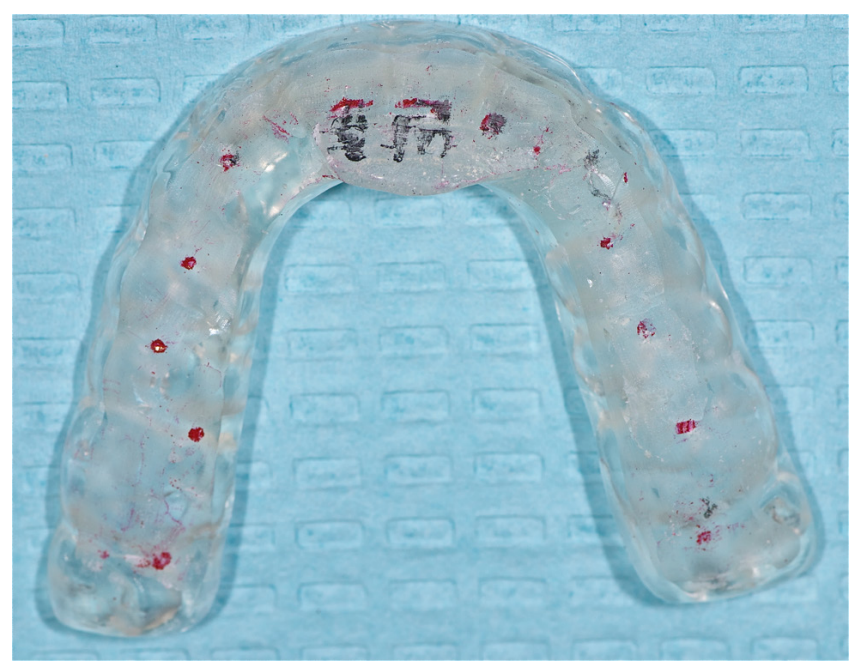

Figure 3 Maxillary anterior repositioning appliances ARA. This is a maxillary ARA adjusted to even bilateral contact with the patient in a protrusive position. The ramp in the anterior is added and retroclined to hold the patient in this position. There is some freedom of movement. It is important that the patient have good posterior support and even contact to minimize any trauma to the mandibular anterior teeth. ARA, anterior repositioning appliance. proponents of ACs feel that the canines should be included because of their particular proprioceptive qualities with no incisal or posterior contact (37). To date, most studies show no additional benefit in comparing ACs with FPs. Furthermore, headache studies have shown that there was minimal effect in improvement of migraine headaches using ACs when compared to low dose tricyclic antidepressant medication (38). There have been both one MAUDE database report and anecdotal evidence to indicate that these appliances even worn just at night can cause occlusal changes with resulting anterior open bite. However, here have been no formal studies on this (Figure 4).

In addition, other studies have indicated that the lack of posterior support with ACs may result in increased joint loading and an increase in joint sounds (39). Given the current evidence, ACs should be used judiciously in patients with overriding muscle pain and no joint pathology. It should also be noted that up to one-third of clinically healthy and quiet temporomandibular joints have disc displacements (40), so this fact should be kept in mind whenever such appliances are being used. To date, there have been no studies specifically evaluating these appliances 

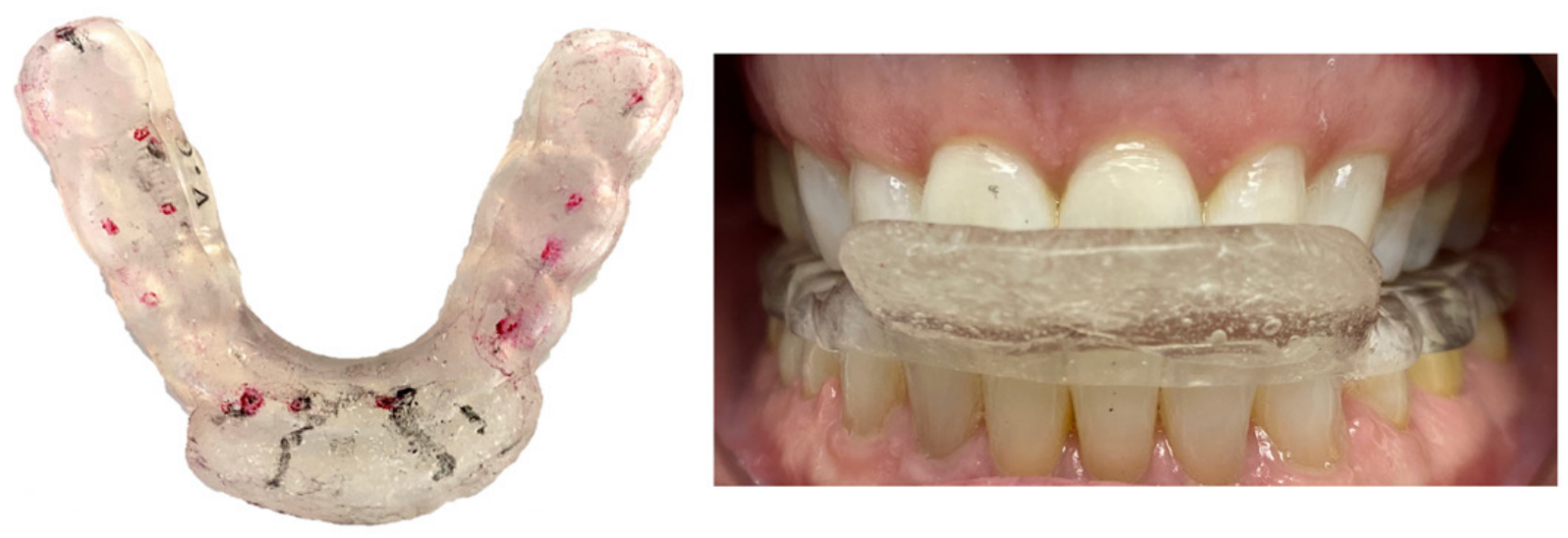

Figure 4 Lower anterior ARA with labial ramp. This OA is adjusted with equal bilateral support. The posterior support also minimizes any trauma to the maxillary anterior teeth. This is shown to demonstrate that a lower flat plane OA can be converted without having to remake new upper OA for the patient. ARA, anterior repositioning appliance; OA, oral appliance.

in patients with significant DC/TMD axis II (behavioral) diagnoses.

\section{Posterior contact appliances (PCA)}

Historically, PCAs have been used in an effort to distract the joints vertically, even though the mandible is a Class III lever which cannot be distracted in that manner. Dr. Harold Gelb (32) has been credited with the concept that TMDs are best managed by positioning the condyle at a specific radiographic point in the glenoid fossa. This position was accomplished using a mandibular appliance (MORA) with occlusal coverage over the posterior teeth only. While this concept may have provided relief for some patients, a common complication with these appliances was that wearing them over extended periods of time resulted in intrusion of the posterior dentition or possible eruption of the open anterior bite.

\section{Neuromuscular OAs}

Neuromuscular OAs are directive (anterior repositioning) and are usually placed over the mandible following 30-45 minute of TENS stimulation (22). Advocates believe that this leads to the mandible being in a directed position where the patient has muscular relaxation determined by surface EMG reading from the muscles of mastication. To date there is no research support for the validity of this "myocentric" position. This device is intended for more than just therapeutic use and is meant to determine jaw position prior to occlusal therapy.

\section{Common errors with the use of $\mathrm{OAs}$}

There are many controversies associated with the process of choosing a particular OA, and clinical errors are often made in their use and application (41). Patient compliance is a huge issue. The most common errors reported by expert clinicians working in orofacial pain clinics is that patients present with ill-fitting OAs that are unstable, too bulky, and poorly adjusted. Some of these appliances have poor retention or are overly tight, creating pressure and tooth discomfort resulting in poor patient acceptance (42). However, hard acrylic OAs can be relieved in the intaglio surface and relined to accommodate a small number of new restorations or even to correct minor errors in processing to improve fit.

Thermoplastic materials are often used to fabricate OAs. While some thermoplastic materials when laminated with hard acrylic may be rigid, many thermoplastic materials used for OAs are resilient, even when laminated with a rigid cameo shell. Other thermoplastic appliances are fabricated in one uniform thickness of material that mimic the anatomy of the underlying occlusal surfaces; due to the posterior envelope of function, these thermoplastic appliances may be easily perforated in the distal segments with normal wear and/or therapeutic adjustment. There is some support in the literature for resilient materials helping to reduce pain palliatively; however, there has also been data showing that soft thermoplastic appliances may increase nocturnal bruxism (28). Resilient thermoplastic appliances may be useful occasionally as temporary appliances because they can often be fabricated in the dental office with 
simple armamentarium; however, adjusting, finishing, and polishing resilient thermoplastic OAs due to the properties of the material itself.

A serious common error in use of OAs is not having standardized protocols to follow the patient for needed adjustments after insertion (depending on diagnosis). Many patients have simply had an OA inserted and then not been followed up. In fact, based on the reports of many orofacial pain patients, OAs are commonly inserted by dentists in some cases by dental auxiliaries with a misunderstanding that they are accurate coming directly from the laboratory. They often may not be refined clinically based on the diagnosis for which they were fabricated.

\section{Proposal for a Controlled study of the flat plane (FP) appliance}

Based on the above discussions and review of this topic, it is reasonable to conclude that the current research base for determining the true value of OAs is not satisfactory. The authors recognize that no single proposal for a new clinical study could totally resolve this situation. However, there are several general research principles that could be applied to produce a properly conducted clinical evaluation of the most commonly used OA: namely, the full mouth flat plane (stabilization) appliance (43). In this next section, a specific list of the experimental conditions and a specific proposal for conducting that study is presented.

As previously discussed, in order to substantiate the use of OAs, the previous heterogeneity in RCTs for OAs, small sample size, lack of control, risk bias control, and multiple methods of data collection and analysis need to be avoided to the extent possible. There are limits to standardization of any OA study design, and this needs to be recognized. The most significant obstacles are consistency of splint design, operator variation, accurate diagnosis, and inclusion and exclusion criteria of subject groups. It may be impossible to double blind these studies, but blind evaluators could be used to assess patient progress and outcomes.

The authors propose that the first basic study be designed to differentiate the outcomes from OA therapy for different DC/TMD diagnoses. Future studies could be conducted to investigate a number of other factors where the past evidence base has showed contradictory findings. The following is a suggestion for such a study.

\section{OA design}

FP appliance would be adjusted meticulously and fitted for patient comfort adjusted in habitual closure. The material should be heat or cold cured methyl methacrylate. Alternatively, a rigid thermoplastic base appliance could be used with laminated acrylic to create the cameo and occluding surface. Researchers should be experienced and proficient in fitting and adjusting OAs, which would involve calibration training. The standard FP should be adjusted with the thinnest available articulating paper (e.g., Accufilm ( $)$ or Shimstock $\left({ }^{\circledR}\right)$ to minimize hinge axis effects. Occlusal contact computer measurements with pressure foil should not be included due to excessive thickness of the foil (100 microns) (44). The subject patients should be recalled at stipulated and consistent intervals.

\section{Subject selection}

Subjects would be selected using the DC/TMD categories and examination protocol (45). In studies with disc displacements, the Wilkes classification could also be used (32). Initial studies should make every attempt to minimize the inclusion of complex diagnoses. It is suggested that initial studies be done on patients with acute symptoms to keep study design as simple as possible. It would be preferable not to have any crossover design.

\section{Controls}

The study should be conducted as a one-modality experiment, comparing FP OA to an untreated control (this has been done in past studies by using subjects who have been waiting for TMD therapy and have had no treatment). Alternatively, the "control" group could be provided with patient education and self-management strategies with no $\mathrm{OA}$, while the treatment group would have those combined with the use of a FP appliance. Psychometric testing is also essential for research subjects according to the DC/TMD research protocol. Obvious axis II findings should be excluded in the original studies. Patients being treated for depression, anxiety, OCD, and co-morbidities such as FMS, rheumatoid arthritis, CFS, should be excluded. The study patients should also have a full complement of teeth, excluding third molars.

\section{Outcome measurement}

Since there are no good objective pain measurements, a visual analog scale (VAS) is the recommended assessment standard. Mandibular range of motion could also be considered as an objective measurement.

\section{Rationale}

It is essential that a baseline efficacy for OA therapy be 
established. Other than the obvious motivation for providing patients with valid evidenced based treatment to manage pain from TMD, it is also an access to care issue. In the United States and other countries with third party payers, coverage for OA therapy is often denied or poorly reimbursed because of lack of evidence (46). If a baseline for OA therapy can be established according to diagnosis, outcomes can be documented to hopefully improve acceptance and reimbursement from third party payers for treatment.

\section{Conclusions}

The ultimate purpose of this paper was to present a current summary of how OAs might be of clinical value for treating various TMD conditions within the DC/TMD framework. After reading the above review of the current evidence in relation to this issue, it would be reasonable to conclude that the answer to that question remains somewhat controversial, and thus is far from being perfectly clear. Nevertheless, it also is reasonable to state that the position taken by some that OAs are of no value at all for treating such patients is not correct. The combination of positive clinical studies and extensive experience in both academic and private clinical settings is more than enough to offset the cynical viewpoint that OAs are simply worthless. As reported above, most of the negative studies that appear to support that type of negative conclusion are seriously flawed in terms of patient selection, diagnostic confusion, poorly controlled conditions, and much more.

Therefore, we the authors believe that the evidence presented in this paper supports the conclusion that OAs are potentially very useful in certain clinical situations, such as inflammatory joint conditions and regional myofascial pain. It is less certain that they are helpful for patients who demonstrate chronic widespread bilateral pain conditions, especially with psychosocial issues overlying their symptoms; but, if conservatively applied, they might be effective in reducing some of the symptoms and signs of those conditions. At worst, they should not be harmful for that cohort of patients. Finally, we recognize that OAs are not likely to help patients who present with certain kinds of longstanding chronic TMDs or who have complex comorbid conditions (e.g., fibromyalgia), and they rarely will enable a patient who has benign clicking TMJs to eliminate their clicking. Therefore, it would not be recommended by us that such patients receive an $\mathrm{OA}$ as part of their TMD management. It is our hope that new researchers can benefit from, modify, and improve our recommendations for new studies so that both the profession and the public can have increased confidence in the use of OAs.

\section{Acknowledgments}

Our appreciation to the following for their input: Gary Klasser DMD Diplomate of Orofacial Pain LSU School of Dental Medicine, Joseph McCain DMD OMS Massachusetts General Hospital, Nigel Shaun Matthews DMD MD OMS UNC Chapel Hill. Funding: None.

\section{Footnote}

Provenance and Peer Review: This article was commissioned by the Guest Editors (Stephen Feinberg and Louis Mercuri) for the series "Temporomandibular Joint Disorders Diagnosis and Management-What Does the Future Hold?" published in Frontiers of Oral and Maxillofacial Medicine. The article has undergone external peer review.

Conflicts of Interest: All authors have completed the ICMJE uniform disclosure form (available at https://fomm. amegroups.com/article/view/10.21037/fomm-20-49/coif). The series "Temporomandibular Joint Disorders Diagnosis and Management-What Does the Future Hold?" was commissioned by the editorial office without any funding or sponsorship. The authors have no other conflicts of interest to declare.

Ethical Statement: The authors are accountable for all aspects of the work in ensuring that questions related to the accuracy or integrity of any part of the work are appropriately investigated and resolved.

Open Access Statement: This is an Open Access article distributed in accordance with the Creative Commons Attribution-NonCommercial-NoDerivs 4.0 International License (CC BY-NC-ND 4.0), which permits the noncommercial replication and distribution of the article with the strict proviso that no changes or edits are made and the original work is properly cited (including links to both the formal publication through the relevant DOI and the license). See: https://creativecommons.org/licenses/by-nc-nd/4.0/.

\section{References}

1. Behr M, Knüttel H, Fanghänel J, et al. The history of 
the concepts in treating craniomandibular dysfunctions using occlusal appliances. A review. Trends in Dentistry 2018;1:101-13.

2. Greene CS, Menchel HF. The Use of Oral Appliances in the Management of Temporomandibular Disorders. Oral Maxillofac Surg Clin North Am 2018;30:265-77.

3. Fouda AAF. No evidence on the effectiveness of oral splints for the management of temporomandibular joint dysfunction pain in both short and long-term follow-up systematic reviews and meta-analysis studies. J Korean Assoc Oral Maxillofac Surg 2020;46:87-98.

4. Riley P, Glenny AM, Worthington HV, et al. Oral splints for temporomandibular disorder or bruxism: a systematic review. Brit Dent J 2020;228:191-7.

5. Al-Moraissi EA, Farea R, Qasem KA, et al. Effectiveness of occlusal splint therapy in the management of temporomandibular disorders: network meta-analysis of randomized controlled trials. Int J Oral Maxillofac Surg 2020;49:1042-56.

6. Katyayan PA, Katayan MH. Efficacy of Appliance Therapy on Temporomandibular Disorder Related Facial Pain and Mandibular Mobility: A Randomized Controlled Study. J Indian Prosthodont Soc 2014;14:251-61.

7. Fricton J, Look JO, Wright E, et al. Systematic review and meta-analysis of randomized controlled trials evaluating intraoral orthopedic appliances for temporomandibular disorders. J Orofac Pain 2010;24:237-54.

8. Nagata K, Maruyama H, Mizuhashi R, et al. Efficacy of stabilisation splint therapy combined with nonsplint multimodal therapy for treating RDC/TMD axis I patients: a randomised controlled trial. J Oral Rehabil 2015;42:890-9.

9. Costa YM, Porporatti AL, Stuginski-Barbosa J, et al. Additional effect of occlusal splints on the improvement of psychological aspects in temporomandibular disorder subjects: A randomized controlled trial. Arch Oral Biol 2015;60:738-44.

10. Giannakopoulos NN, Rauer AK, Hellmann D, et al. Comparison of device-supported sensorimotor training and splint intervention for myofascial temporomandibular disorder pain patients. J Oral Rehabil 2018;45:669-676.

11. Al-Ani MZ, Davies SJ, Gray RJ, et al. Stabilisation splint therapy for temporomandibular pain dysfunction syndrome. Cochrane Database Syst Rev 2004;(1):CD002778.

12. De la Torre Canales G, Manfredini D, Grillo CM, et al. Therapeutic effectiveness of a combined counseling plus stabilization appliance treatment for myofascial pain of the jaw muscles: A pilot study. Cranio 2017;35:180-6.

13. Qvintus V, Suominen AL, Huttunen J, et al. Efficacy of stabilisation splint treatment on facial pain-1-year followup. J Oral Rehabil 2015;42:439-46.

14. Wahlund K, Nilsson IM, Larsson B, et al. Treating temporomandibular disorders in adolescents: a randomized, controlled, sequential comparison of relaxation training and occlusal appliance therapy. J Oral Facial Pain Headache 2015;29:41-50.

15. Kuzmanovic Pficer J, Dodic S, Lazic V, et al. Occlusal stabilization splint for patients with temporomandibular disorders: Meta-analysis of short and long term effects. PLoS One 2017;12:e0171296.

16. Rosar JV, Barbarosa TS, Diaz IOV, et al. Effect of interocclusal appliance on bite force, sleep quality, salivary cortisol levels and signs and symptoms of temporomandibular dysfunction in adults with sleep bruxism. Arch Oral Biol 2017;82:62-70.

17. Schiffman E, Ohrbach R, Truelove E, et al. Diagnostic Criteria for Temporomandibular Disorders (DC/TMD) for Clinical and Research Applications: recommendations of the International RDC/TMD Consortium Network and Orofacial Pain Special Interest Group. J Oral Facial Pain Headache 2014;28:6-27.

18. Klasser GD, Greene CS. Oral appliances in the management of temporomandibular disorders. Oral Surg Oral Med Oral Pathol Oral Radiol Endod 2009;107:212-23.

19. Okeson JP. Occlusal appliance therapy. In: Okeson JP. Management of Temporomandibular Disorders and Occlusion. St. Louis: Mosby, 2008:492-4.

20. Simmons HC. Guidelines for anterior repositioning Appliance therapy for the management of craniofacial pain and TMD. Cranio 2005;23:300-5.

21. Dawson PE. Functional Occlusion from TMJ to Smile Design. 2nd ed. St. Louis MO: Mosby, 2007:36-7.

22. Jankelson B. Neuromuscular aspects of occlusion. Dent Clin North Am 1979;23:157-68.

23. Greene CS. "The Ball on the Hill": A new perspective on TMJ functional anatomy. Orthod Craniofac Res 2018;21:170-4.

24. Milam SB, Schmitz JP. Molecular biology of temporomandibular joint disorders. J Oral Maxillofac Surg 1995;53:1448-54.

25. Milam SB, Zardentata G, Schmitz JB. Oxidative stress and degenerative temporomandibular joint disease: A proposed hypothesis. J Oral Maxillofac Surg 1998;56:214-23.

26. Baş B, Aksoy A, Atmaca E, et al. Effect of occlusal splint on interleukin 6, malondialdehyde and 
8-hydroxydeoxyguanosine levels in the synovial fluid of patients with temporomandibular disorders. Int J Oral Maxillofac Surg 2019;48:1558-63.

27. Raphael KG, Marbach JJ. Widespread pain and the effectiveness of oral splints in myofascial face pain. J Am Dent Assoc 2001;132:305-16.

28. Okeson JP. The effects of hard and soft occlusal splints on nocturnal bruxism. JADA 1987;114:788-91.

29. Cruz-Reyes RA, Martinez-Aragon I, Guerro-Arias RE, et al. Influence of occlusal stabilization splints and soft occlusal splints on the electromyographic pattern, in basal state and at the end of six weeks treatment in patients with bruxism. Acta Odontol Latinoam 2011;24:66-74.

30. Dao TT, Lavigne GJ. Oral splints: the crutches for temporomandibular orders and bruxism? Crit Rev Oral Biol Med 1998;9:345-61.

31. Whitney CW, Von Korff M. Regression to the mean in treated vs not treated chronic pain. Pain 1992;50:281-5.

32. Gelb ML, Gelb H. Mandibular orthopedic repositioning therapy. Cranio Clin Int 1991;1:81-98.

33. Wilkes CH. Internal derangements of the temporomandibular joint. Pathological variations. Northwest Dent 1990;69:25-32.

34. Tecco S, Festa F, Salini V, et al. Treatment of joint pain and joint noises associated with a recent TMJ internal derangement: a comparison of an anterior repositioning splint, a full-arch maxillary stabilization splint, and an untreated control group. Cranio 2004;22:209-19.

35. Shankland WE. Nociceptive trigeminal inhibitiontension suppression system: a method of preventing migraine and tension headaches. Compend Contin Educ Dent 2001;22:1075-1080. [corrected and republished 2002;23:105-8.]

36. Stapelmann H, Turp J. The NTI-tss device for the therapy of bruxism, temporomandibular disorders, and headache Where do we stand? A qualitative systematic review of the

doi: 10.21037/fomm-20-49

Cite this article as: Menchel HF, Greene CS, Huff KD. Intraoral appliances for temporomandibular disorders: what we know and what we need to know. Front Oral Maxillofac Med 2021;3:6. literature. BMC Oral Health 2008;8:22.

37. Crout DK. Anatomy of an occlusal splint. Gen Dent 2017;65:52-9.

38. Bruno MAD, Krymchantowski AV. Amitryptyline and intraoral devices for migraine prevention: a randomized comparative trial. Arq Neuropsiquiatr 2018;76:213-8.

39. Conti PC, Corrêa AS, Lauris JR, et al. Management of painful temporomandibular joint clicking with different intraoral devices and counseling: a controlled study. J Appl Oral Sci 2015;23:529-35.

40. Tallents RH, Katzberg RW, Murphy W, et al. Magnetic resonance imaging findings in asymptomatic volunteers and symptomatic patients with temporomandibular disorders. J Prosthet Dent 1996;75:529-33.

41. Widmalm SE. Use and abuse of bite splints. Compend Contin Educ Dent 1999;20:249-54, 256, 258-9; quiz 260.

42. Moufti MA, Lilico JT, Wassell RW. How to make a wellfitting stabilization splint. Dent Update 2007;34:398-400, 402-4, 407-8.

43. Rehm DD, Mainieri VC, Saueressig AC, et al. Effects of the bite splint 15-day treatment termination in patients with temporomandibular disorder with a clinical history of sleep bruxism: a longitudinal single-cohort study.Oral Surg Oral Med Oral Pathol Oral Radiol 2012;114:740-8.

44. Sharma A, Rahul GR Poduval ST, et al. History of materials used for recording static and dynamic occlusal contact marks: A literature review J Clin Exp Dent 2013;5:e48-53.

45. Ohrbach R, Gonzalez Y, List T, et al. Diagnostic Criteria for Temporomandibular Disorders: DC/TMD Clinical Examination Protocol. Version January 6, 2014. Available online: www.rdc-international.org

46. Bond EM, Mackey S. Temporomandibular Disorders: Priorities for Research and Care (2020). Washington, DC: The National Academies Press. Available online: http:// nap.edu/25652 